\title{
A $C(K)$ BANACH SPACE WHICH DOES NOT HAVE THE SCHROEDER-BERNSTEIN PROPERTY
}

\author{
PIOTR KOSZMIDER
}

\begin{abstract}
We construct a totally disconnected compact Hausdorff space $K_{0}$ which has clopen subsets $K_{2} \subseteq K_{1} \subseteq K_{0}$ such that $K_{0}$ is homeomorphic to $K_{2}$ and hence $C\left(K_{0}\right)$ is isometric as a Banach space to $C\left(K_{2}\right)$ but $C\left(K_{0}\right)$ is not isomorphic to $C\left(K_{1}\right)$. This gives two nonisomorphic Banach spaces of the form $C(K)$ which are isomorphic to complemented subspaces of each other (even in the above strong isometric sense), providing a solution to the SchroederBernstein problem for Banach spaces of the form $C(K) . K_{0}$ is obtained as a particular compactification of the pairwise disjoint union of a sequence of $K \mathrm{~s}$ for which $C(K)$ s have few operators.
\end{abstract}

\section{INTRODUCTION}

If $X, Y$ are Banach spaces, then $X \sim Y$ will mean that they are isomorphic, and $X \stackrel{c}{\hookrightarrow} Y$ that $Y$ has a complemented subspace isomorphic to $X$. A. Pełczyński has proved (see [19, 3]) that if two Banach spaces $X, Y$ satisfy $X \sim X^{2}, Y \sim Y^{2}$, then the following version of the Schroeder-Bernstein theorem holds for them:

$$
\text { If } X \stackrel{c}{\hookrightarrow} Y \text { and } Y \stackrel{c}{\hookrightarrow} X \text {, then } X \sim Y \text {. }
$$

The problem whether this holds in general, without demanding that $X$ and $Y$ are isomorphic to their squares has been known as the Schroeder-Bernstein problem for Banach spaces (3]). After several decades it has been solved in the negative by T. Gowers in 11. In this paper we give quite different construction of a Banach space which solves this problem in the negative which additionally is a classical Banach space of real valued continuous functions on a compact Hausdorff space with the supremum norm.

One of the main ingredients of Gowers' solution was the use of Banach spaces $X_{G M}$, obtained by Gowers himself and Maurey ([12]), with few operators in the sense that each bounded, linear operator on it is of the form $\lambda I d+S$ where $S$ is strictly singular and $\lambda$ is a scalar. The space of [11] is an "exotic" completion of $c_{00}\left(X_{G M} \oplus X_{G M}\right)$ where the norm in the sum is defined depending on the choice of a basic sequence in $X_{G M}$. Also the space $X_{G M}$ has quite a complex definition of the norm. Other examples of Banach spaces which solve the Schroeder-Bernstein

The author was partially supported by Polish Ministry of Science and Higher Education research grant N N201 386234 and Junta de Andalucía and FEDER grant P06-FQM-01438. The research leading to this paper was conducted while the author was visiting Universidad de Granada in the spring of 2009. He would like to thank Professors Miguel Martín and Javier Merí for their hospitality and fruitful discussions. 
problem in the negative were also based on spaces like $X_{G M}$ and were constructed by Gowers and Maurey [13, Galego ([6], 7], 8]) or Galego and Ferenczi [9].

When we deal with Banach spaces of continuous functions on a compact Hausdorff space $K$, we face, however, the simplest possible norm, the supremum norm and nontrivial examples are obtained through nontrivial compact $K$ s. In [15] we considered Banach spaces of continuous functions with few operators in the sense that any operator on them is of the form $g I d+S$ where $g \in C(K)$ and $S$ is weakly compact (equivalently here, a strictly singular operator) or such that the adjoint of any operator is of the form $g I d+S$ where $g$ is a Borel bounded function and $S$ is weakly compact on the dual. The latter construction and its connected modification was used in 15 to obtain results analogous to some of the results of Gowers and Maurey but in the class of $C(K)$ Banach spaces, like indecomposable Banach spaces or spaces nonisomorphic to their hyperplanes. The former construction was obtained in [15] under the assumption of the continuum hypothesis but later was modified in [21] or 24] to avoid this assumption. Of course the former form of the operator is simpler and we will make use of $C(K)$ Banach spaces with few operators in this sense. For our purpose the construction of 24 is the most convenient because its $K$ is perfect, separable and totally disconnected and the Boolean algebra $C l o p(K)$ of all clopen subsets of $K$ has a dense subalgebra with many automorphisms. On the other hand by the results of [25] the approach where we deal with spaces with few adjoint operators in the above sense would have to work as well but would be formally more complicated.

The general plan of the construction and what should work follows [11 with $X_{G M}$ replaced by a $C(K)$ with few operators. However, all this must be achieved in the $C(K)$-environment, that is through an appropriate compactification of the topological disjoint sum of compact $K_{i}$ s rather than the definition of a norm. Note, also, that if $K$ is infinite and metrizable then $C(K)$ is isomorphic to its square, so we must deal with nonseparable $C(K)$ s. So, the realization of this general plan is quite far from [11. Another complication is that our spaces have few operators in a different sense that those of [12], this seems to be an obstacle which caused that we were unable to obtain spaces which are isomorphic to their cube but not isomorphic to their square as in the paper [11. If there could be such spaces of the form $C(K)$ still remains an open problem.

The main idea is to define a Banach space $X_{+}$as a $C\left(K_{+}\right)$where $K_{+}$is an appropriate compactification of the pairwise disjoint sum of clopen subsets $K_{n}=$ $K_{1, n} \cup K_{2, n}$ for $n \in \mathbb{N}$, such that all operators from $C\left(K_{n}\right)$ into itself are of the form $f I d+S$ where $f \in C\left(K_{n}\right)$ and $S$ is weakly compact and all $K_{n}$ are pairwise homeomorphic. It follows that all operators from $C\left(K_{i, n}\right)$ into $C\left(K_{3-i, m}\right)$ are weakly compact, where $i=1,2$ and $n, m \in \mathbb{N}$.

$Y_{+}=\left\{f \in X_{+}: f \mid K_{1,0}=0\right\} \sim C\left(K_{+} \backslash K_{1,0}\right)$. It is clear that $Y_{+}$is complemented in $X_{+}$and the compactification is defined in such a way that $\left\{f \in X_{+}: f \mid K_{1}=0\right\}$ is a subspace of $Y_{+}$isomorphic to $X_{+}$and clearly complemented in $Y_{+}$. Thus we only face the proof of the fact that $X_{+}$and $Y_{+}$are not isomorphic.

Although $C\left(K_{i, n}\right)$ and $C\left(K_{3-i, m}\right)$ are quite incomparable (there are only weakly compact and so strictly singular operators among them), we exploit the fact that both of the algebras of all clopen sets of $K_{1, n}$ and $K_{2, n}$ have isomorphic dense subalgebras. This allows us to compare $f_{i} \in K_{i, n}$ for $i=1,2$ using an automorphism of these dense subalgebras. Finally the compactification $K_{+}$is defined in such a way 
that each $f \in C\left(K_{+}\right)$can be identified with a sequence of functions $f_{n} \in C\left(K_{n}\right)$ such that $f_{n} \mid K_{1, n}$ and $f_{n} \mid K_{2, n}$ get "closer" to each other (in a sense defined using the dense subalgebras and an automorphism between them) when $n$ tends to infinity. This construction and the notation used to deal with it is described in Section 3.

The point of the proof that $X_{+}$is not isomorphic to $Y_{+}$is to prove that any isomorphism $T$ between these spaces would create infinitely many "bumps" i.e., $T\left(f_{n}\right) \mid K_{1, m(n)}$ s and $T\left(f_{n}\right) \mid K_{2, m(n)}$ s would not get closer to each other when $n$ and some $m(n)$ tend to infinity. This would give $T(f)$ outside of $X_{+}$leading to a contradiction with the existence of such an isomorphism. At least this is what happens when we look at the shift of the part of $X_{+}$corresponding to $\bigcup_{n \in \mathbb{N}} K_{1, n}$ which should not be a well-defined operator on $X_{+}$. It turns out that all hypothetic isomorphisms from $Y_{+}$to $X_{+}$would share this behaviour and so there are none of them. This argument is the subject of the last section where a strengthening of a well-known fact that $\left\{x \in K: T^{*}\left(\delta_{x}\right)(\{x\}) \neq 0\right\}$ is at most countable, if $T$ is weakly compact is also proved (6.2).

To be able to detect these bumps one approximate an operator $T: X_{+} \rightarrow X_{+}$ by a matrix of multiplications by continuous functions. This approximation is developed in Section 5 which relies on Section 4 where we prove general properties of operators on $X_{+}$. Some of the ideas of Section 4 which we use for dealing with compactifications of disjoint $K_{i}$ s where for each $i \in \mathbb{N}$ the space $C\left(K_{i}\right)$ has few operators were developed in [17] and [16].

The next Section 2 gathers some classical results about $C(K)$ s and some of their corollaries needed in the rest of the paper.

\section{Some fundamental Results on $C(K)$ SPACES}

In this section we recall some fundamental results of Bessaga, Grothendieck, Pełczyński and Rosenthal concerning the Banach spaces $C(K)$. We will do quote them in the form most convenient for the sake of this paper, also we make some simple corollaries.

Theorem 2.1. [4](Cor. 2) Let $E$ be a Banach space and $X$ be either $l_{\infty}$ or $c_{0}$ and let $T: X \rightarrow E$ be a linear continuous operator. Then exactly one of the two possibilities holds:

(1) $T\left(e_{n}\right) \rightarrow 0$

(2) There is an infinite subset $M \subseteq \mathbb{N}$ such that $T \mid X(M)$ is an isomorphism.

Theorem 2.2. 22] (Thm 1.3) Let $B$ be a Banach space and $X$ an injective space. Let $T: X \rightarrow B$ be an operator such that there exists a subspace $A$ of $X$ isomorphic to $c_{0}(\Gamma)$, with $T \mid A$ an isomorphism. Then there exists a subspace $Y$ of $X$ isomorphic to $l_{\infty}(\Gamma)$ with $T \mid Y$ an isomorphism.

Definition 2.3. Let $K$ be a Hausdorff compact space. Suppose that $\left(f_{n}\right)$ is a sequence from $C(K)$. We say that it generates a copy of $c_{0}$ if and only if it is a basic sequence which is equivalent to the standard basis of $c_{0}$.

Definition 2.4. Let $K$ be a Hausdorff compact space. We say that $f, g \in C(K)$ are disjoint if and only if $f g=0$. 
Fact 2.5. Let $K$ be a Hausdorff compact space and $\varepsilon>0$. Suppose that $\left(f_{n}\right)$ is a bounded, pairwise disjoint sequence of functions such that $\left\|f_{n}\right\|>\varepsilon>0$ for each $n \in \mathbb{N}$. Then $\left(f_{n}\right)$ generates a copy of $c_{0}$.

Fact 2.6. Let $K$ be a Hausdorff compact space. Suppose that $\left(f_{n}\right)_{n \in \mathbb{N}}$ is a sequence of functions such that $\left(f_{n}\right)_{n \in \mathbb{N}}$ generates a copy of $c_{0}$ and suppose that $a_{n} \subseteq K$ are clopen sets such that $\left\|f_{n} \chi_{a_{n}}\right\|>\varepsilon$ for some $\varepsilon>0$. Then there is an infinite $M \subseteq \mathbb{N}$ such that $\left(f_{n} \chi_{a_{n}}\right)_{n \in M}$ generates a copy of $c_{0}$

Proof. Let $T: c_{0} \rightarrow C(K)$ be an isomorphism such that $T\left(1_{n}\right)=f_{n}$. Note that for each $x \in K$ we have

$$
\sum_{n \in \mathbb{N}} \alpha_{n} f_{n} \chi_{a_{n}}(x)=\sum_{n \in \mathbb{N}} \alpha_{n}^{*}(x) f_{n}(x),
$$

where $\alpha_{n}^{*}(x)=\alpha_{n}$ if $x \in a_{n}$ and $\alpha_{n}^{*}(x)=0$ otherwise, and so, such an $\left(\alpha_{n}^{*}(x)\right)_{n \in N}$ belongs to $c_{0}$. For every $\varepsilon>0$ and every $k_{1}<k_{2}$ from $\mathbb{N}$ there is an $x \in K$ such that

$$
\begin{gathered}
\left\|\sum_{n=k_{1}}^{k_{2}} \alpha_{n} f_{n} \chi_{a_{n}}\right\| \leq\left\|\sum_{n=k_{1}}^{k_{2}} \alpha_{n}^{*}(x) f_{n}\right\|+\varepsilon \leq \\
\leq\left\|T \left|\left\|\mid \alpha_{k_{1}}^{*}(x), \ldots, \alpha_{k_{2}}^{*}(x)\right\|_{c_{0}}+\varepsilon \leq\|T\|\left\|\alpha_{k_{1}}, \ldots, \alpha_{k_{2}}\right\|_{c_{0}}+\varepsilon .\right.\right.
\end{gathered}
$$

So, if $\left(\alpha_{n}\right) \in c_{0}$, we have that $\left\|\alpha_{k_{1}}, \ldots, \alpha_{k_{2}}\right\|_{c_{0}}$ goes to zero if $k_{1}$ and $k_{2}$ go to infinity, hence we may conclude that $\sum_{n \in \mathbb{N}} \alpha_{n} f_{n} \chi_{a_{n}}$ converges uniformly and so the limit is continuous. A similar argument shows that $\left\|\sum_{n \in \mathbb{N}} \alpha_{n} f_{n} \chi_{a_{n}}\right\| \leq\left\|T\left|\left\|\mid\left(\alpha_{n}\right)\right\| \|_{c_{0}}\right.\right.$. So we may conclude that

$$
T^{\prime}\left(\left(\alpha_{n}\right)\right)=\sum_{n \in \mathbb{N}} \alpha_{n} f_{n} \chi_{a_{n}}
$$

is a bounded linear operator defined on $c_{0}$ and into $C(K)$. Now we can use 2.1 since $\left\|T^{\prime}\left(1_{n}\right)\right\|=\left\|f_{n} \chi_{a_{n}}\right\|>\varepsilon$.

Theorem 2.7. 20](Thm. 1) A linear bounded operator on $T: C(K) \rightarrow C(K)$ is weakly compact if and only if it is strictly singular.

Theorem 2.8. 2] A linear bounded operator on $T: C(K) \rightarrow C(K)$ is weakly compact if and only if for every bounded pairwise disjoint sequence $\left(f_{n}\right) \subseteq C(K)$ we have $T\left(f_{n}\right) \rightarrow 0$.

Theorem 2.9. 22] Suppose that $\varepsilon>0$ and for each $n, k \in \mathbb{N}$ we have non-negative $m_{n k} \in \mathbb{R}$ and $\sum_{n \in \mathbb{N}} m_{n k}<\varepsilon$ for each $k \in \mathbb{N}$. Then for each $\delta>0$ there is an infinite $M \subseteq \mathbb{N}$ such that

$$
\sum_{n \in M \backslash\{k\}} m_{n k}<\delta
$$

for each $k \in M$.

Corollary 2.10. Suppose $\left(f_{n}\right)$ is a bounded pairwise disjoint sequence of elements of a $C(K)$. Suppose $\left(\zeta_{n}\right)_{n \in \mathbb{N}}$ is a bounded sequence in $C^{*}(K)$ and let $\varepsilon>0$. There is an infinite $M \subseteq \mathbb{N}$ such that whenever $M^{\prime} \subseteq M$ and the supremum $f_{M^{\prime}}=$ $\sup _{n \in M^{\prime}} f_{n}$ exists in $C(K)$, then $\left|\zeta_{k}\left(f_{M^{\prime}}\right)-\zeta_{k}\left(f_{k}\right)\right|<\varepsilon$ if $k \in M^{\prime}$ and $\left|\zeta_{k}\left(f_{M^{\prime}}\right)\right|<\varepsilon$ if $k \in M \backslash M^{\prime}$ 
Proof. Let $\mu_{k}$ be the Radon measure on $K$ corresponding to $\zeta_{k}$. Define $m_{n k}=$ $\left|\mu_{k}\right|\left(f_{n}\right)$. Apply the Rosenthal lemma 2.9 for $\delta=\varepsilon$ obtaining an infinite $M_{1} \subseteq M$ such that then $\sum_{n \in M_{1} \backslash\{k\}}\left|\zeta_{k}\left(f_{n}\right)\right|<\varepsilon$ if $k \in M_{1}$. Thus, it is enough to obtain an infinite $M \subseteq M_{1}$ such that whenever $M^{\prime} \subseteq M$ and the supremum $f_{M^{\prime}}=\sup _{n \in M^{\prime}} f_{n}$ exists in $C(K)$, then for each $k \in \mathbb{N}$

$$
\sum_{n \in M^{\prime}} \zeta_{k}\left(f_{n}\right)=\zeta_{k}\left(f_{M^{\prime}}\right)
$$

Note that

$$
\sum_{n \in M^{\prime}} \zeta_{k}\left(f_{n}\right)=\int \sum_{n \in M^{\prime}} f_{n} d \mu_{k}
$$

and $\zeta_{k}\left(f_{M^{\prime}}\right)=\int f_{M^{\prime}} d \mu_{k}$ where $\sum_{n \in M^{\prime}} f_{n}$ is taken pointwise and is possibly not in $C(K)$. Let $\left\{\mathbb{N}_{\xi}: \xi<\omega_{1}\right\}$ be a family of infinite sets of $\mathbb{N}$ whose pairwise intersections are finite. If none of them works as $M$, there is a $k \in \mathbb{N}$ and there are infinite $b_{\xi} \subseteq \mathbb{N}_{\xi}$ for uncountably many $\xi \in \omega_{1}$ such that

$$
\int\left(f_{b_{\xi}}-\sum_{n \in b_{\xi}} f_{n}\right) d \mu_{k} \neq 0
$$

but this is impossible since the Borel functions which we integrate above are pairwise disjoint as shown in the claim of 5.2. of [15].

Theorem 2.11. 1] Let $X$ be a Banach space $\left(x_{n}\right) \subseteq X$ be a basic sequence and $\left(x_{n}^{*}\right) \subseteq X^{*}$ a sequence biorthogonal to $\left(x_{n}\right)$. If $\left(y_{n}\right) \subseteq X$ fulfills the condition

$$
\sum_{n \in \mathbb{N}}\left\|x_{n}-y_{n}\right\|\left\|x_{n}^{*}\right\|<\delta<1,
$$

Then $\left(y_{n}\right)$ is a basic sequence and $\left(x_{n}\right)$ and $\left(y_{n}\right)$ are equivalent.

\section{The COnstruction And the notation}

The Stone functor from the category of Boolean algebras and homomorphisms into the category of compact Hausdorff spaces with continuous functions will be denoted $S$. Thus $S(A)$ is the Stone space of the algebra $A$ and $S(h): S(B) \rightarrow S(A)$ is the continuous mapping induced by a homomorphism $h: A \rightarrow B$ of Boolean algebras and is given by $S(h)(x)=h^{-1}(x)$. If $a$ is an element of a Boolean algebra $A$, the basic clopen set $\{u \in S(A): a \in u\}$ of the Stone space of $A$ corresponding to $a$ will be denoted by $[a]$. The Boolean algebra of clopen subsets of a compact space $K$ is denoted by $C l o p(K)$. S $(C l o p(K))$ will be identufied with $K$ and $C l o p(S(A)$ with $A$. For more on the Stone duality see Chapter 3 of [14].

Lemma 3.1. There is an infinite Boolean algebra $A$ whose Stone space $K$ is a sum $K_{1, *} \cup K_{2, *}$ for $K_{1, *}$ and $K_{2, *}$ disjont and clopen which satisfies the following:

(1) $K$ is separable (compact totally disconnected) perfect space,

(2) every operator on $C(K)$ is of the form $T=f I d+S$ where $f \in C(K)$ and $S$ is weakly compact, 
(3) $C(K)$ contains no copy of $l_{\infty}$,

(4) there is a dense subalgebra $B \subseteq A$ such that $K_{1, *}, K_{2, *} \in B$ and an automorphism $j: B \rightarrow B$ such that $j\left(K_{3-i, *}\right)=K_{i, *}$ for $i=1,2$ and $j^{2}=I d_{B}$,

(5) suppose that $\left(a_{n}\right)$ is a sequence of clopen and pairwise disjoint subsets of $K$. There is an infinite $M \subseteq \mathbb{N}$ such that there is in $A$ the supremum $\sup _{n \in M} a_{n}=a$.

Proof. Let $K$ be the Stone space of the algebra $A$ obtained in Chapter 3 of [24]. By 3.6.1, 3.6.2., 3.6.3., and the fact that $K$ has property $\left(K^{\prime}\right)(3.3 .1$.) we obtain (1) without the perfectness, (2) and (5). (3) follows from 2.4. of [15] because every space which contain $l_{\infty}$ must have its hyperplanes isomorphic to itself. So, we are left with showing that there is a dense subalgebra $B$ and its automorphism $j$ as above. $B$ will be a free Boolean algebra with $2^{\omega}$ independent generators. The algebra $A$ includes the algebra $B$ of clopen subsets of $\{0,1\}^{2^{\omega}}$ and is included the algebra of regular open subsets of $\{0,1\}^{2^{\omega}}$ hence $B$ is dense in $A$ (see [24. p. 57). As $B$ is a free Boolean algebra, it is clear that there is an automorphism and $K_{1, *}$, $K_{2, *}$ as required and that $K$ is prefect.

Remark 3.2. In the literature there are several constructions of spaces $C(K)$ where all operators are of the form $T=f I d+S$ where $f \in C(K)$ and $S$ is weakly compact: those obtained using some special set theoretic assumptions in Section 6 of [15], or in [5] and having some additional properties or without any additional set-theoretic assumptions spaces of [21] or [24]. The spaces of Section 6 of [15] or of [5] have countable subalgebras B satysfying the above theorem. The space 21] is only presented in the connected version and it is unclear to us if its totally disconnected version would have some natural dense subalgebras with appropriate automorphisms. The separability of $K$ from 24 is not used in any argument in this paper but it shows that a $C(K)$ solution to the Schroeder-Bernstein problem could be a subspace of $l_{\infty}$. We conjecture that also the space of Section 3 of [15] obtained without any special set-theoretic assumptions can be used to obtain the main result of this paper, the results of 25] support this conjecture, however it seems that it would complicate the details. Thus it seems that the space of [24] is the most optimal for our purpose.

Let $A, B, K, K_{1, *}, K_{2, *}, j$ be as in 3.1, moreover let

- $C=\{b \in B: j(b)=b\}=\left\{b \cup j(b): b \in B,[b] \subseteq K_{1, *}\right\}=\{j(b) \cup b: b \in$ $\left.B,[b] \subseteq K_{2, *}\right\}$

- $L$ be the Stone space of $C$,

- $\tau=S(\subseteq): K \rightarrow L$ is the canonical surjection, where $\subseteq: C \rightarrow A$ is the inclusion.

- $Z \equiv C(L)$ considered as a subspace of $C(K)$ i.e., $Z=\{f \circ \tau: f \in C(L)\}$.

Remark 3.3. Note that $\tau$ identifies, among others, the pairs $x, y$ of points of $K_{1, *}$ and $K_{2, *}$ respectively such that $S(j)(x \cap B)=y \cap B$. So in particular, for each $x \in K_{i, *}$ there is $y \in K_{3-i, *}$ such that $\tau(x)=\tau(y)$.

As $K$ is separable we may w.l.o.g. assume that $A$ is a subalgebra of $\wp(\mathbb{N})$. For $n \in \mathbb{N}$ let

- $\mathbb{N}_{n} \mathrm{~s}$ be pairwise disjoint copies of $\mathbb{N}$, 
- $A_{n}$ s be the copies of $A$ in $\wp\left(\mathbb{N}_{n}\right)$,

- $K_{n} \mathrm{~s}$ be the (pairwise disjoint) Stone spaces of $A_{n} \mathrm{~s}$,

- $K_{1, n}$ and $K_{2, n}$ be the copies of $K_{1, *}$ and $K_{2, *}$ in $K_{n}$,

- $K^{-n}=\bigcup_{i \leq n} K_{i}$,

- $B_{n}$ be the copy of $B$ in $A_{n} \subseteq \wp\left(\mathbb{N}_{n}\right)$,

- $C_{n}$ be the copy of $C$ in $A_{n} \subseteq \wp\left(\mathbb{N}_{n}\right)$,

- $L_{n}$ be the Stone space of $C_{n}$,

- $\tau_{n}=S\left(\subseteq_{n}\right): K_{n} \rightarrow L_{n}$ be the canonical surjection, where $\subseteq_{n}: C_{n} \rightarrow A_{n}$ is the inclusion.

- $j_{n}$ be the copy of $j$ on $B_{n}$.

- $i_{n, *}: K \rightarrow K_{n}, i_{*, n}: K_{n} \rightarrow K, i_{m, n}: K_{n} \rightarrow K_{m}$ be homeomorphisms preserving the above mentioned objects respectively,

- $h_{n, *}: L \rightarrow L_{n}, h_{*, n}: L_{n} \rightarrow L, h_{m, n}: L_{n} \rightarrow L_{m}$ be homeomorphisms.

We will consider the following Boolean algebras:

- $D_{\infty}=\left\{a \subseteq \bigcup_{n \in \mathbb{N}} \mathbb{N}_{n}: \forall n \in \mathbb{N} \quad a \cap \mathbb{N}_{n} \in A_{n}\right\}$,

- $D_{0}=\left\{a \in D_{\infty}: \exists m \in \mathbb{N}\left(\forall n>m a \cap \mathbb{N}_{n}=\emptyset\right)\right.$ or $\left.\left(\forall n>m a \cap \mathbb{N}_{n}=\mathbb{N}_{n}\right)\right\}$,

- $D_{+}=\left\{a \in D_{\infty}: \exists m \in \mathbb{N} \forall n>m\right.$ a $\left.\cap \mathbb{N}_{n} \in C_{n}\right\}$.

In other words, $D_{\infty}$ is the product algebra of $A_{n} \mathrm{~s}, D_{0}$ is the direct sum algebra of $A_{n}$ s, and finally $D_{+}$is the algebra of those elements of $D_{\infty}$ whose coordinates eventually belong to $C_{n}$.

Let

- $K_{\infty}, K_{0}, K_{+}$be the Stone spaces of $D_{\infty}, D_{0}$, and $D_{+}$respectively.

And finally let

- $X_{\infty}=C\left(K_{\infty}\right), X_{+}=C\left(K_{+}\right), Z_{n} \equiv C\left(L_{n}\right)$ considered as a subspace of $C\left(K_{n}\right)$ i.e., $Z_{n}=\left\{f \circ \tau_{n}: f \in C\left(L_{n}\right)\right\}$.

- $X_{0}=C_{0}\left(K_{0}\right)=\left\{f \in C\left(K_{0}\right): f(\infty)=0\right\}$, where $\infty$ is the only ultrafilter of $D_{0}$ which does not contain any $\mathbb{N}_{n}$.

- $K^{+n}=K_{+} \backslash K^{-n}$.

We also will need the following notation for some natural projections and inclusions, let

- $P_{n}: C\left(K_{+}\right) \rightarrow C\left(K_{n}\right)$ be the restriction to $K_{n}$.

- $I_{n}: C\left(K_{n}\right) \rightarrow C\left(K_{+}\right)$be the extension from $K_{n}$ by zero function.

- $P_{-n}: C\left(K_{+}\right) \rightarrow C\left(K^{-n}\right)$ be the restriction to $K^{-n}$.

- $I_{-n}: C\left(K^{-n}\right) \rightarrow C\left(K_{+}\right)$be the extension from $K^{-n}$ by zero function.

- $P_{+n}: C\left(K_{+}\right) \rightarrow C\left(K^{+n}\right)$ be the restriction to $K^{+n}$.

- $I_{+n}: C\left(K^{+n}\right) \rightarrow C\left(K_{+}\right)$be the extension from $K^{+n}$ by zero function.

The Stone-Weierstrass theorem which implies that the functions which assume only finitely many values on clopen sets from the algebra $A$ are dense in the $C(K)$ where $K$ is the Stone space of $A$, gives the idea about which functions belong to the spaces $X_{0}, X_{+}, X_{\infty}$. In particular we have the following three descriptions :

Lemma 3.4.

$$
X_{\infty} \equiv\left\{\left(f_{n}\right)_{n \in N} \in \Pi_{n \in \mathbb{N}} C\left(K_{n}\right):\left(\left\|f_{n}\right\|\right)_{n \in \mathbb{N}} \text { is bounded }\right\} .
$$

The norm is the supremum norm.

Lemma 3.5.

$$
X_{0} \equiv\left\{\left(f_{n}\right)_{n \in N} \in \Pi_{n \in \mathbb{N}} C\left(K_{n}\right): \lim _{n \rightarrow \mathbb{N}}\left\|f_{n}\right\|=0\right\} .
$$


The norm is the supremum norm.

For $f \in C\left(K_{n}\right)$ denote by $d_{n}(f)$ the distance between $f$ and the subspace $Z_{n} \subseteq$ $C\left(K_{n}\right)$. Thus we have:

\section{Lemma 3.6.}

$$
X_{+} \equiv\left\{\left(f_{n}\right)_{n \in N} \in \Pi_{n \in \mathbb{N}} C\left(K_{n}\right):\left(\left\|f_{n}\right\|\right)_{n \in \mathbb{N}} \text { is bounded and } \lim _{n \rightarrow \infty} d_{n}\left(f_{n}\right)=0\right\} .
$$

The norm is the supremum norm.

We will often denote elements of the above spaces using the above representations i.e., as a sequence $\left(f_{n}\right)$. Then, under the appropriate identification we have $X_{0} \subseteq$ $X_{+} \subseteq X_{\infty}$ which will be used as well.

Definition 3.7. For $x, y \in K_{n}$ we say that $x \bowtie y$ if and only if $\tau_{n}(x)=\tau_{n}(y)$.

Lemma 3.8. Suppose that $f \in C\left(K_{n}\right)$. Then

$$
d_{n}(f)=\frac{\sup \left\{|f(x)-f(y)|: x, y \in K_{n}, x \bowtie y\right\}}{2} .
$$

Proof. Let $s$ be the supremum from the lemma. If $g \in Z_{n}$, then clearly $g(x)=g(y)$ for any $x, y$ such that $x \bowtie y$ we obtain $d_{n}(f) \geq s / 2$.

Now let $f \in C\left(K_{n}\right)$ and $\delta>s$. It is possible to obtain a clopen finite partition $U_{1}, \ldots, U_{k}$ of $L_{n}$ such that $\operatorname{diam}\left(f\left[\tau_{n}^{-1}\left[U_{m}\right]\right]\right)<\delta$ for each $m=1, \ldots, k$. This follows from the fact that $\operatorname{diam}\left(f\left[\tau_{n}^{-1}[\{t\}]\right]\right) \leq s$ for each $t \in L_{n}$ and the continuity of $f$ as well as the compactness of the spaces involved. Now define $g \in Z_{n}$ as a function which is constant on each $\tau_{n}^{-1}\left[U_{m}\right]$ and assumes on it the arithmetic average of the extrema of the values of $\left.f\left[\phi^{-1}\left[U_{m}\right]\right]\right)$. This way $\|f-g\| \leq \delta / 2$ and hence $d_{n}(f) \leq s / 2$.

Corollary 3.9. Suppose that $g \in Z_{n}$ and that $f \in C\left(K_{n}\right)$ is arbitrary such that $\|f-g\|<\varepsilon$. Suppose that $x, y \in K_{n}$ are such that $x \bowtie y$. Then $|f(x)-f(y)|<2 \varepsilon$.

Proof. Follows directly from the previous lemma.

Corollary 3.10. Suppose that $\left(f_{n}\right)_{n \in \mathbb{N}} \in X_{+}$and $x_{n}, y_{n} \in K_{n}$, are such that $x_{n} \bowtie y_{n}$. Then $\left|f_{n}\left(x_{n}\right)-f_{n}\left(y_{n}\right)\right|$ converges to 0 as $n$ tends to infinity.

Proof. Follows directly from the previous corollary and from Lemma 3.6 .

Finally we will need the following notation:

- $Y_{0}=\left\{\left(f_{n}\right) \in X_{0}: f \mid K_{1,0}=0\right\}$

- $Y_{+}=\left\{\left(f_{n}\right) \in X_{+}: f \mid K_{1,0}=0\right\}$.

Lemma 3.11. $Y_{+}$is complemented in $X_{+}$and $X_{+}$contains a complemented subspace isometric to $Y_{+}$. Both projections of norm one.

Proof. Clearly $X_{+}=C\left(K_{+}\right) \equiv C\left(K_{1,0}\right) \oplus C\left(K_{2,0}\right) \oplus\left\{\left(f_{n}\right) \in X_{+}: f_{1}=0\right\}$ and $\left\{\left(f_{n}\right) \in X_{+}: f_{1}=0\right\}$ is isometric to $X_{+}$while $C\left(K_{2,0}\right) \oplus\left\{\left(f_{n}\right) \in X_{0}: f_{1}=0\right\}$ is isometric to $Y_{+}$.

The rest of this paper is devoted to the proof that $Y_{+}$is not isomorphic to $X_{+}$. 


\section{Operators ON $X_{+}$}

First, we will define two operators which will serve to illustrate several phenomena in Proposition 4.2 and Remarks 4.8 and 5.8 ,

Definition 4.1. Fix a pairwise disjoint sequence $\left(g_{n}\right)$ in $C(K)$ such that $\left\|g_{n}\right\|=1$ and fix a dense countable subset $\left\{x_{n}: n \in \mathbb{N}\right\}$ of $K$ and for each $n \in \mathbb{N}$ fix $\theta_{n} \in C^{*}(K)$ such that $\left\|\theta_{n}\right\|=1$ and such that $\theta_{n} \mid Z_{n}=0$.

- Define $\Lambda: C(K) \rightarrow X_{+}$by $\Lambda(f) \mid K_{n}=f\left(x_{n}\right) \chi_{K_{n}}$ for $f \in C(K)$, and

- define $\Theta: X_{+} \rightarrow C(K)$ by $\Theta\left(\left(f_{n}\right)\right)=\sum_{n \in \mathbb{N}} \theta_{n}\left(f_{n}\right) g_{n}$.

Proposition 4.2. $\Theta$ and $\Lambda$ are well-defined linear bounded operators. The image of $\Theta$ is isomorphic to $c_{0}$, in particular $X_{+}$is not a Grothendieck space and so, it has a complemented copy of $c_{0}$.

Proof. The only nontrivial part of the first statement is whether $\Theta\left(\left(f_{n}\right)\right)$ is always in $C(K)$ for any $\left(f_{n}\right) \in X^{+}$. It is enough to note that $\theta_{n}\left(f_{n}\right)$ s tend to zero. But for each $n \in \mathbb{N}$ there is a $z_{n} \in Z_{n}$ such that $\left\|f_{n}-z_{n}\right\|$ s go to zero. Then using the fact that $\theta_{n} \mid Z_{n}=0$ we have

$$
\left|\theta_{n}\left(f_{n}\right)\right|=\left|\theta_{n}\left(f_{n}-z_{n}\right)+\theta_{n}\left(z_{n}\right)\right| \leq|| \theta_{n}|||| f_{n}-z_{n} \|
$$

which goes to zero. So, the last statement follows as well (see [23] 5.1, 5.3).

Note that $X=\left\{\left(f_{n}\right) \in X_{+}: \forall n \in \mathbb{N} f_{n} \mid K_{n}\right.$ is constant $\}$ is a complemented in $X_{+}$copy of $l_{\infty}$. Thus using it and the operators $\Theta$ and $\Lambda$ we may construct many nontrivial operators from $X^{+}$into $X^{+}$which have nothing to do with any multiplications. It was already noted in [17] that nevertheless dealing with this kind of spaces we have strong tools (see for example 4.7) to analyze all the operators.

Lemma 4.3. Suppose that $T: C(K) \rightarrow X_{+}$is a linear bounded operator. Let $\left(f_{m}\right)_{m \in \mathbb{N}}$ be a bounded sequence of pairwise disjoint functions in $C(K)$. Then

$$
\forall \varepsilon \exists k \forall n>k \forall m \quad d_{n}\left(T\left(f_{m}\right) \mid K_{n}\right) \leq \varepsilon .
$$

Proof. We may w.l.o.g. assume that the norms of the sequence of functions are bounded by 1 . Suppose that the lemma is false. Let $\varepsilon>0$ be such that for every $k \in \mathbb{N}$ there is $n>k$ and $m_{k} \in \mathbb{N}$ such that

$$
d_{n}\left(T\left(f_{m_{k}}\right) \mid K_{n}\right)>\varepsilon .
$$

Hence we can choose increasing $n_{k}$ 's such that

$$
d_{n_{k}}\left(T\left(f_{m_{k}}\right) \mid K_{n_{k}}\right)>\varepsilon \text {. }
$$

Moreover as $T\left(f_{m_{k}}\right) \in X_{+}$by 3.6 we must have that $m_{k}$ s assume infinitely many values and so we may assume that

$$
d_{n_{k}}\left(T\left(f_{m_{k}}\right) \mid K_{n_{k}}\right)>\varepsilon .
$$

and $m_{k}$ s are increasing.

By 3.8 there are points $x_{k}, y_{k} \in K_{n_{k}}$ such that $x_{k} \bowtie y_{k}$ and for each $k \in \mathbb{N}$

$$
\left|T\left(f_{m_{k}}\right)\left(x_{k}\right)-T\left(f_{m_{k}}\right)\left(y_{k}\right)\right|>2 \varepsilon \text {. }
$$


Let

$$
\mu_{k}=T^{*}\left(\delta_{x_{k}}-\delta_{y_{k}}\right) .
$$

So we have that $\left|\int f_{m_{k}} d \mu_{k}\right|>2 \varepsilon$. Since $K$ is totally disconnected we can choose pairwise disjoint clopen $\left[a_{m_{k}}\right]$ included in the supports of $f_{m_{k}}$ such that

$$
\left|\mu_{k}\left(\left[a_{m_{k}}\right]\right)\right|>\varepsilon .
$$

Now we use 2.10 for the pairwise disjoint bounded sequence $\left(\chi_{\left[a_{m_{k}}\right]}\right)_{k \in \mathbb{N}}, \varepsilon / 2$ and the functionals equal to the measures $\mu_{k}$ and then 3.1 (5) obtaining an infinite $M \subseteq \mathbb{N}$ such that there is the supremum $a=\sup _{k \in M} a_{m_{k}}$ in $C l o p(K)$ and $\left|\mu_{k}(a)-\mu_{k}\left(a_{m_{k}}\right)\right|<\varepsilon / 2$ for each $k \in M$. However this implies that $\mid T\left(\chi_{[a]}\right)\left(x_{k}\right)-$ $T\left(\chi_{[a]}\right)\left(y_{k}\right) \mid>\varepsilon / 2$ for each $k \in M$ which by 3.10 implies that $T\left(\chi_{[a]}\right)$ is not in $X_{+}$, a contradiction.

Definition 4.4. Let $f \in X_{+}$. We say that $f$ is $\left(C_{n}\right)$-simple if for every $n \in \mathbb{N}$ the function $f \mid K_{n}$ assumes only finitely many values on clopen sets from $C_{n}$.

Lemma 4.5. Suppose that $\left(f_{m}\right)$ is a sequence of elements of $X_{+}$are such that

(1) $f_{m} s$ are $\left(C_{n}\right)$-simple for each $m \in \mathbb{N}$

(2) $f_{m} \mid K_{-m}=0$

(3) $\left(f_{m}\right)$ generates a copy of $c_{0}$.

Suppose that $T: X_{+} \rightarrow C(K)$ is a bounded linear operator. Then $\left\|T\left(f_{m}\right)\right\| s$ converge to 0 .

Proof. We will find an injective subspace of $X_{+}$which contains all the $f_{m}$ 's and then assuming that the lemma is false we will apply 2.1 and 2.2 to obtain a contradiction with 3.1 (3). Let $E_{n}$ be the Boolean algebra of subsets of $K_{n}$ generated by the preimages under the functions $f_{m}$ where $m \in \mathbb{N}$. Note that it is finite as almost all the functions $f_{m}$ are zero on $K_{n}$ and the remaining functions are $\left(C_{n}\right)$-simple. Consider the Boolean algebra

$$
E=\left\{a \in D_{\infty}: \forall n \in \mathbb{N} \quad a \cap \mathbb{N}_{n} \in E_{n}\right\},
$$

and note that it is a complete Boolean algebra and hence the Banach space $V$ of all continuous functions which are in the closure of finite linear combinations of characteristic functions of elements of $E$ is included in $X_{+}$, is injective and that $f_{m} \in V$ for each $m \in \mathbb{N}$.

Now, if $\left\|T\left(f_{m}\right)\right\|$ s do not converge to 0 , then by 2.1 we may w.l.o.g. assume that $T$ is an isomorphism on the copy of $c_{0}$ generated by $\left(f_{m}\right)$. Finally apply 2.2 to conclude that $C\left(K_{n}\right)$ contains a copy of $l_{\infty}$ which gives a contradiction with 3.1 (3).

Lemma 4.6. Suppose that $S: C(K) \rightarrow X_{+}$and $T: X_{+} \rightarrow C(K)$ and $\left(f_{m}\right)$ is a pairwise disjoint sequence in $C(K)$ which generates a copy of $c_{0}$ such that for every $n \in \mathbb{N}$ we have

$$
\left\|T \circ I_{-n} \circ P_{-n} \circ S\left(f_{m}\right)\right\| \rightarrow 0,
$$

whenever $m \rightarrow \infty$. Then $\left\|T\left(S\left(f_{m}\right)\right)\right\| \rightarrow 0$. 
Proof. Assume that the lemma is false i.e., that $\left\|T\left(S\left(f_{m}\right)\right)\right\|$ s do not converge to 0 . Then $\left\|S\left(f_{m}\right)\right\|$ s do not converge to 0 and so by 2.1 we may w.l.o.g assume that $\left(S\left(f_{m}\right)\right)_{m \in \mathbb{N}}$ generates a copy of $c_{0}$.

By the assumption that $\left\|T \circ I_{-n} \circ P_{-n} \circ S\left(f_{m}\right)\right\| \rightarrow 0$ and the hypothesis that $\left\|T\left(S\left(f_{m}\right)\right)\right\|$ s do not converge to 0 for every $n \in \mathbb{N}$ we may choose $m_{n} \in \mathbb{N}$ such that $\left\|T \circ I_{+n} \circ P_{+n} \circ S\left(f_{m_{n}}\right)\right\|$ s are separated from 0 and so also $P_{+n}\left(S\left(f_{m_{n}}\right)\right)$ s are separated from zero.

Applying [2.6] we may choose a strictly increasing sequence $\left(n_{k}\right)$ such that $n_{k}>k$ and such that $P_{+n_{k}} \circ S\left(f_{m_{n_{k}}}\right)$ generate a copy of $c_{0}$.

Now we will look for some basic sequence equivalent to $\left(P_{+n_{k}}\left(\left(S\left(f_{m_{n_{k}}}\right)\right)\right)\right.$. Let $\rho>1$ be a bound for norms of a sequence biorthogonal to $\left(P_{+n_{k}}\left(S\left(f_{m_{n_{k}}}\right)\right)\right)$.

By 4.3 we can thin-out the sequence $\left(n_{k}\right) \subseteq \mathbb{N}$ so that

$$
\left|d_{n}\left(P_{n}\left(P_{+n_{k}}\left(S\left(f_{m_{n_{k}}}\right)\right)\right)\right)\right|<\frac{1}{2^{k+2} \rho}
$$

holds for all $n, k \in \mathbb{N}$

By the above and the Weierstrass-Stone theorem we can find $\left(g_{k}\right)$ s which are $\left(C_{n}\right)$-simple, satisfy $P_{-n}\left(g_{n}\right)=0$ for each $n \in \mathbb{N}$ and such that

$$
\left\|g_{k}-I_{+n_{k}} \circ P_{+n_{k}} \circ S\left(f_{m_{n_{k}}}\right)\right\|<\frac{1}{2^{k+2} \rho},
$$

which means by the Bessaga-Pełczyński criterion 2.11 that $\left(g_{k}\right)$ generates a copy of $c_{0}$. Thus by 4.5 we have that $\left\|T\left(g_{k}\right)\right\|$ s converge to 0 . But $\| T\left(g_{k}-I_{+n_{k}} \circ P_{+n_{k}} \circ\right.$ $S\left(f_{m_{n_{k}}}\right) \|$ s also converge to 0 which means that $T \circ I_{+n_{k}} \circ P_{+n_{k}} \circ S\left(f_{m_{n_{k}}}\right)$ s converge to 0 , a contradiction with the choice of $m_{n}$ 's.

Corollary 4.7. Suppose that $S: C(K) \rightarrow X_{+}$and $T: X_{+} \rightarrow C(K)$ are such that for every $n \in \mathbb{N}$ either

(1) $P_{-n} \circ S$ is weakly compact or

(2) $T \circ I_{-n}$ is weakly compact.

Then $T \circ S: C(K) \rightarrow C(K)$ is weakly compact.

Proof. Let $\left(f_{m}\right)$ be a bounded pairwise disjoint sequence in $C(K)$. We will use 2.8, and so we need to prove that $(T \circ S)\left(f_{m}\right)$ converges to zero. By 2.1 we may w.l.o.g. assume that $\left(f_{m}\right)$ generates a copy of $c_{0}$. By the hypothesis of the corollary $T \circ I_{-n} \circ P_{-n} \circ S$ are weakly compact for each $n \in \mathbb{N}$ and so by 2.8, $T \circ I_{-n} \circ P_{-n} \circ S\left(f_{m}\right)$ converges to zero when $m$ tends to infinity and $n \in \mathbb{N}$ is fixed. So, we may apply 4.6 to conclude that $(T \circ S)\left(f_{m}\right)$ converges to zero and that $T \circ S$ is weakly compact.

Remark 4.8. Suppose that $\Lambda: C(K) \rightarrow X^{+}$and $\Theta: X^{+} \rightarrow C(K)$ are as in 4.1. Note that $P_{-n} \circ \Lambda$ and $\Theta \circ P_{-n}$ are finite dimensional and so weakly compact for all $n \in \mathbb{N}$ but neither $\Lambda$ nor $\Theta$ are weakly compact. This shows that the composition cannot be replaced by a single operator in the above results. Note that $\Theta \circ \Lambda=0$. 


\section{The MATRIX OF MULTIPLICATIONS OF AN OPERATOR}

Lemma 5.1. Suppose that $K^{\prime}$ and $K^{\prime \prime}$ are homeomorphic perfect compact spaces and $i: K^{\prime \prime} \rightarrow K^{\prime}$ is a homeomorphism between them. Let $\phi, \psi \in C\left(K^{\prime}\right)$ and $S$ : $C\left(K^{\prime}\right) \rightarrow C\left(K^{\prime \prime}\right)$ be a weakly compact operator. Suppose that for every $f \in C\left(K^{\prime}\right)$ we have

$$
(\phi f) \circ i=(\psi f) \circ i+S(f) .
$$

Then $S=0$ and $\phi=\psi$.

Proof. Suppose that $\psi \neq \psi$ and choose a clopen $U \subseteq K^{\prime}$ or and $\varepsilon>0$ such that $|(\phi-\psi)| U \mid>\varepsilon$. Let $f_{n}$ s be pairwise disjoint functions of norm one whose supports are included in $U$. They exist since $K^{\prime}$ is perfect. Note that $\left\|(\phi-\psi) f_{n}\right\| \not \rightarrow 0$ and so $\left\|\left[(\phi-\psi) f_{n}\right] \circ i\right\| \not \rightarrow 0$ since $i$ is onto $K^{\prime}$. This contradicts the fact that $S\left(f_{n}\right) \rightarrow 0$ by 2.8 , Now $S$ must be zero as well.

Suppose that $T: X_{+} \rightarrow X_{+}$is an operator. By 3.1 (2) for each $n, m \in \mathbb{N}$ there are continuous functions $\phi_{m, n}^{T} \in C\left(K_{n}\right)$ and weakly compact operators $S_{m, n}^{T}$ : $C\left(K_{n}\right) \rightarrow C\left(K_{m}\right)$ such that for each $f \in C\left(K_{n}\right)$

$$
T(f) \mid K_{m}=\left[\phi_{m, n}^{T} f\right] \circ i_{n, m}+S_{m, n}^{T}(f) .
$$

We will skip the superscript $T$, if it is clear from the context. In this section we analyze the matrix $\left(\phi_{m, n}^{T}\right)_{m, n \in \mathbb{N}}$ for an operator $T$ on $X_{+}$. Note that Lemma 5.1 implies that such a decomposition of an operator is unique.

Lemma 5.2. Let $T: X_{+} \rightarrow X_{+}$be an operator. Let $n \in \mathbb{N}$ be fixed. The sequence $\left(\phi_{m, n}^{T}\right)_{m \in \mathbb{N}}$ converges to 0 .

Proof. Fix $n \in \mathbb{N}$. If the lemma is false, there are points $x_{m} \in K_{n}$ and an $\varepsilon>0$ such that $\left|\phi_{m, n}\left(x_{m}\right)\right|>\varepsilon$ for $m$ 's from an infinite set $M_{1} \subseteq \mathbb{N}$. We may w.l.o.g. assume that $x_{m} \in K_{1, n}$ or $x_{m} \in K_{2, n}$ for all $m \in \mathbb{N}$. Say $x_{m} \in K_{1, n}$, the other case is analogous. By the continuity of $\phi_{m, n}$ and the fact that $K_{n}$ is perfect we can choose pairwise disjoint open sets $U_{m} \subseteq K_{1, n}$ such that $\left|\phi_{m, n}(x)\right|>\varepsilon$ holds for every $x \in U_{m}$

For $m \in \mathbb{N}$ fixed let $\alpha_{k, m}: K_{n} \rightarrow \mathbb{R}$ be pairwise disjoint characteristic functions whose supports $V_{k, m}$ are included in $U_{m}$. Now we use a characterization 2.8 of weakly compact operators on $C(K)$ spaces, concluding for every $m \in M_{1}$ that $\left\|S_{m, n}\left(\alpha_{k, m}\right)\right\|$ converges to zero when $k$ converges to infinity. So, for each $m \in M_{1}$ we can choose $k(m) \in \mathbb{N}$ such that

$$
\left\|S_{m, n}\left(\alpha_{k(m), m}\right)\right\|<\varepsilon / 5 .
$$

The definitions of $S_{m, n}$ and the behaviour of $\phi_{m, n}$ on $U_{m}$ imply that for $x \in$ $i_{m, n}\left[V_{k(m), m}\right]$ we have

and for all $x \in K_{2, m}$

$$
\left|T\left(\alpha_{k(m), m}\right)(x)\right|>4 \varepsilon / 5
$$

$$
\left|T\left(\alpha_{k(m), m}\right)(x)\right|<\varepsilon / 5
$$

for each $m \in M_{1}$. For $m \in M_{1}$ pick $y_{m} \in i_{m, n}\left[V_{k(m), m}\right]$ and $z_{m} \in K_{2, m}$ such that $y_{m} \bowtie z_{m}$ (see 3.3). Consider $\zeta_{m}=T^{*}\left(\delta_{y_{m}}\right) \mid K_{n}$ and $\theta_{m}=T^{*}\left(\delta_{z_{m}}\right) \mid K_{n}$ (restrictions of measures on $K_{+}$to $\left.K_{n}\right)$ as functionals on $C\left(K_{n}\right)$. Now apply 2.9 twice, obtaining 
an infinite $M_{2} \subseteq M_{1}$ such that whenever $M \subseteq M_{2}$ is an infinite set such that there is the supremum $\sup _{m \in M} \alpha_{k(m), m}=\alpha$ then

$$
\begin{aligned}
& \left|\zeta_{m}(\alpha)-\zeta_{m}\left(\alpha_{k(m), m}\right)\right|<\varepsilon / 5, \\
& \left|\theta_{m}(\alpha)-\theta_{m}\left(\alpha_{k(m), m}\right)\right|<\varepsilon / 5 .
\end{aligned}
$$

for $m \in M$. Since $\left|\zeta_{m}\left(\alpha_{k(m), m}\right)\right|>4 \varepsilon / 5$ and $\left|\theta_{m}\left(\alpha_{k(m), m}\right)\right|<\varepsilon / 5$ in terms of $T$ we obtain that for $m \in M$ we have

$$
\begin{aligned}
& \left|T(\alpha)\left(z_{m}\right)\right|>3 \varepsilon / 5 \\
& \left|T(\alpha)\left(y_{m}\right)\right|<2 \varepsilon / 5
\end{aligned}
$$

Of course the above supremus $\alpha$ exist in $C\left(K_{n}\right)$ by 3.1 (5) As $T(\alpha)$ is in $X_{+}$and $y_{m} \bowtie z_{m}$ this contradicts 3.10 and completes the proof of the lemma.

Lemma 5.3. Suppose that $T: X_{+} \rightarrow X_{+}$is a linear bounded operator. Suppose that $m \in \mathbb{N}, F \subseteq \mathbb{N}$ is a finite set such that $\left\|\sum_{n \in F}\left|\phi_{m, n}^{T} \circ i_{n, m}\right|\right\|>\varepsilon$. Then there is $f \in C\left(\bigcup_{n \in F} K_{n}\right)$ which is $\left(C_{n}\right)$-simple and of norm one such that $\left\|T(f) \mid K_{m}\right\|>\varepsilon$.

Proof. Let $x \in K_{m}$ be such that $\sum_{n \in F}\left|\phi_{m, n}^{T} \circ i_{n, m}\right|(x)>\varepsilon$. Either $x \in K_{1, m}$ or $x \in K_{2, m}$. Say $x \in K_{1, m}$, the other case is analogous. By the continuity of $\phi_{m, n}$ and the fact that $K_{n}$ is perfect we can find clopen $U \subseteq K_{1, m}$ such that $\sum_{n \in F}\left|\phi_{m, n} \circ i_{n, m}(x)\right|>\varepsilon+\varepsilon^{\prime}$ holds for every $x \in U$ and some $\varepsilon^{\prime}>0$ and such that no $\phi_{m, n}$ for $n \in F$ changes its sign on $i_{n, m}[U]$ (if $\phi_{m, n}(x)=0$ we may remove $n$ from $F$ ). Let $V_{l} \subseteq U \subseteq K_{1, m}$ for $l \in \mathbb{N}$ be pairwise disjoint. By the density of $B_{m}$ in $\operatorname{Clop}\left(K_{1, m}\right)$ we may choose $V_{l}$ s to be in $B_{m}$. Let

$$
\alpha_{n, l}=\delta_{n} \chi_{i_{n, m}[V(l)]}
$$

where $\delta_{n}= \pm 1$ and its sign is the same as $\phi_{m, n}$ on $i_{n, m}[U]$ so

$$
\sum_{n \in F}\left(\phi_{m, n} \alpha_{n, l}\right) \circ\left(i_{n, m}(x)\right)>\varepsilon+\varepsilon^{\prime} .
$$

for each $x \in V_{l}$ and each $l \in \mathbb{N}$.

Let $W_{l}=j\left[V_{l}\right] \subseteq K_{2, m}$ and let $\beta_{n, l}=\delta_{n} \chi_{i_{n, m}\left[W_{l}\right]}$ Then, for each $l \in \mathbb{N}$

$$
f_{l}=\sum_{n \in F} \alpha_{n, l}+\beta_{n, l}
$$

is $\left(C_{n}\right)$-simple, $f_{l} \in C\left(\bigcup_{n \in F} K_{n}\right)$ and

$$
\sum_{n \in F}\left(\phi_{m, n} f_{l}\right)\left(i_{n, m}(x)\right)>\varepsilon+\varepsilon^{\prime} .
$$

for each $x \in V_{l}$. Moreover $f_{l}$ s are pairwise disjoint.

Now we use a characterization 2.8 of a weakly compact operators on $C(K)$ spaces, so we have that $\left\|\sum_{n \in F} S_{m, n}\left(\alpha_{n, l}+\beta_{n, l}\right)\right\|$ converges to zero when $l$ converges to infinity. So, there is an $l_{0} \in \mathbb{N}$ such that such that

$$
\left\|\sum_{n \in F} S_{m, n}\left(\alpha_{n, l_{0}}+\beta_{n, l_{0}}\right)\right\|<\varepsilon^{\prime}
$$

So, for $x \in V_{l}$ we have

$$
\left|T\left(f_{l_{0}}\right)(x)\right| \geq\left|\sum_{n \in F}\left(\phi_{m, n} f_{l_{0}}\right)\left(i_{n, m}(x)\right)\right|-\| \sum_{n \in F} S_{m, n}\left(\alpha_{n, l_{0}}+\beta_{n, l_{0}}\right)|| \geq \varepsilon
$$

and hence $\left\|T(f) \mid K_{m}\right\|>\varepsilon$ for $f=f_{l_{0}}$. 
Lemma 5.4. Let $T: X_{+} \rightarrow X_{+}$be an operator. Let $m \in \mathbb{N}$ be fixed. The series $\sum_{n \in \mathbb{N}}\left|\phi_{m, n}^{T} \circ i_{n, m}\right|$ converges uniformly.

Proof. Fix $m \in \mathbb{N}$. If the series does not converge uniformly, then there is $\varepsilon>0$ and there are strictly increasing $n_{k} \in \mathbb{N}$ and $x_{k} \in K_{m}$ for $k \in \mathbb{N}$ such that

$$
\sum_{n_{k} \leq n<n_{k+1}}\left|\phi_{m, n}\left(i_{n, m}\left(x_{k}\right)\right)\right|>\varepsilon .
$$

We may w.l.o.g. assume that $k<n_{k}$.

By 5.3 we can find $f_{k} \in C\left(\bigcup_{n_{k} \leq n<n_{k+1}} K_{n}\right)$ which are $\left(C_{n}\right)$-simple and of norm one such that $\left\|T\left(f_{k}\right)\right\|>\varepsilon$. As supports of $f_{k}$ s are disjoint they generate a copy of $c_{0}$ by 2.5 . So we obtain a contradiction with 4.5 .

Lemma 5.5. Let $T: X_{+} \rightarrow X_{+}$be an operator and $f=\left(f_{n}\right) \in X_{+}$. The series $\sum_{n \in \mathbb{N}}\left[\phi_{m, n}^{T} f_{n}\right] \circ i_{n, m}$ converges uniformly to a function $g \in C\left(K_{m}\right)$ satisfying $\|g\| \leq$ $\|T||\| \mid f \|$.

Proof. Note that for each $x \in K_{m}$

$$
\begin{gathered}
\left|\sum_{n_{k} \leq n<n_{k+1}}\left[\phi_{m, n} f_{n}\right]\left(i_{n, m}(x)\right)\right| \leq\|f\|\left(\sum_{n_{k} \leq n<n_{k+1}}\left|\phi_{m, n}\left(i_{n, m}(x)\right)\right|\right) \leq \\
\leq\left\|f\left|\|\| \sum_{n_{k} \leq n<n_{k+1}}\right| \phi_{m, n} \circ i_{n, m}\right\|,
\end{gathered}
$$

and so by Lemma 5.4 the partial sums of $\sum_{n \in \mathbb{N}}\left[\phi_{m, n}^{T} f_{n}\right] \circ i_{n, m}$ satisfy the uniform Cauchy condition and so $\sum_{m \in \mathbb{N}}\left[\phi_{n, m}^{T} f_{n}\right] \circ i_{n, m}$ is uniformly convergent. Call the limit $g \in C\left(K_{m}\right)$.

For the proof of the second part of the lemma, note that if $\|g\|>\|T|\||| f\|$, for some $f \in X_{+}$, then by the above inequalities $\sum_{1 \leq n \leq n_{1}}\left|\phi_{n, m}\left(i_{n, m}(x)\right)\right|>\|T\|$ but this would give an $f^{\prime} \in C\left(\bigcup_{1 \leq n \leq n_{1}} K_{n}\right)$ of norm one such that $\left\|T\left(f^{\prime}\right)\right\|>\|T\|$ by 5.3. a contradiction.

Lemma 5.6. Let $T: X_{+} \rightarrow X_{+}$be a bounded linear operator. There is a bounded linear operator $\Pi^{T}: X_{0} \rightarrow X_{0}$ which satisfies

$$
\Pi^{T}(f) \mid K_{m}=\left[\phi_{m, n}^{T} f\right] \circ i_{n, m}
$$

for $f \in C\left(K_{n}\right)$ and whose norm is not bigger than $\|T\|$.

Proof. Let $f \in X_{0}$ and $f_{n}=f \mid K_{n}$. Fix $n \in \mathbb{N}$, by 5.5 for every $m \in \mathbb{N}$ the series $\sum_{n \in \mathbb{N}}\left[\phi_{m, n} f_{n}\right] \circ i_{n, m}$ converges uniformly and its norm is not bigger than $\|T\|\|f\|$. So for $f=\left(f_{n}\right) \in X_{0}$ we can define $\Pi^{T}(f)$ by requiring

$$
\Pi^{T}(f) \mid K_{m}=\left[\sum_{n \in \mathbb{N}} \phi_{m, n}^{T} f_{n}\right] \circ i_{n, m}
$$

which agrees with the condition of the lemma for $f \in C\left(K_{n}\right)$. It is a bounded operator into $X_{\infty}$ whose norm is not bigger than $\|T\|$ by 5.4. However, its restriction to any $C\left(K_{n}\right)$ is into $X_{0}$ by Lemma 5.2 so on $X_{0}$, it is into $X_{0}$.

Lemma 5.7. Let $T, R: X_{+} \rightarrow X_{+}$be any operators and $m, n \in \mathbb{N}$. The operators from $C\left(K_{n}\right)$ into $C\left(K_{m}\right)$ given by

$$
\text { - } P_{m}\left(\Pi^{T}-T\right) R I_{n} \text {, }
$$


- $P_{m}\left(\Pi^{R}-R\right) T I_{n}$,

- $P_{m}\left(\Pi^{T}-T\right)\left(\Pi^{R}-R\right) I_{n}$,

- $P_{m}\left(\Pi^{R}-R\right)\left(\Pi^{T}-T\right) I_{n}$

- $P_{m} R\left(\Pi^{T}-T\right) I_{n}$

- $P_{m} T\left(\Pi^{R}-R\right) I_{n}$,

are all weakly compact.

Proof. Note that for each $k \in \mathbb{N}$ the operators

$$
\begin{aligned}
{\left[P_{m}\left(\Pi^{T}-T\right)\right] I_{-k} } & =\sum_{l \leq k} S_{m, l}^{T} \\
{\left[P_{m}\left(\Pi^{R}-R\right)\right] I_{-k} } & =\sum_{l \leq k} S_{m, l}^{R} \\
P_{-k}\left[\left(\Pi^{T}-T\right) I_{n}\right] & =\sum_{l \leq k} S_{l, n}^{T} \\
P_{-k}\left[\left(\Pi^{R}-R\right) I_{n}\right] & =\sum_{l \leq k} S_{l, n}^{R}
\end{aligned}
$$

are all weakly compact because the right hand sides are finite sums of weakly compact operators. So by 4.7 we may conclude the lemma.

Remark 5.8. If $\Lambda: C(K) \rightarrow X^{+}$and $\Theta: X^{+} \rightarrow C(K)$ are as in 4.1 then they decompose so that all $\phi_{n, *}^{\Lambda}$ and $\phi_{*, m}^{\Theta}$ are zero. This proves that the approximation of a single operator $T$ by $\Pi^{T}$ is not always modulo a weakly compact operator.

Corollary 5.9. Let Suppose that $T, R: X_{+} \rightarrow X_{+}$are such operators that $R \circ T=$ $0_{C\left(K_{1,0}\right)} \oplus I d_{Y_{+}}$and $T \circ R=I d_{X_{+}}$. Then we have $\Pi^{R} \Pi^{T}=0_{C\left(K_{1,0}\right)} \oplus I d_{Y_{0}}$ and $\Pi^{T} \Pi^{R}=I d_{X_{0}}$

Proof. Note that we have

$$
\begin{gathered}
\Pi^{T} \Pi^{R}=\left(\Pi^{T}-T+T\right)\left(\Pi^{R}-R+R\right)= \\
\left(\Pi^{T}-T\right)\left(\Pi^{R}-R\right)+\left(\Pi^{T}-T\right) R+T\left(\Pi^{R}-R\right)+T R
\end{gathered}
$$

and

$$
\begin{gathered}
\Pi^{R} \Pi^{T}=\left(\Pi^{R}-R+R\right)\left(\Pi^{T}-T+T\right)= \\
=\left(\Pi^{R}-R\right)\left(\Pi^{T}-T\right)+\left(\Pi^{R}-R\right) T+R\left(\Pi^{T}-T\right)+R T
\end{gathered}
$$

So by Lemma 5.7 we have

$$
P_{m} \Pi^{T} \Pi^{R} I_{n}=S_{m, n}^{\prime}+P_{m} I_{n}
$$

for all $m, n \in \mathbb{N}$ where $S_{m, n}^{\prime} \mathrm{s}$ are all weakly compact and

$$
P_{m} \Pi^{R} \Pi^{T} I_{n}=S^{\prime \prime}{ }_{m, n}+P_{m} I_{n}
$$

for all $n, m \in \mathbb{N} \backslash\{0\}$ where $S^{\prime \prime}{ }_{m, n}$ are all weakly compact. Moreover

$$
P_{0} \Pi^{R} \Pi^{T} I_{0}=S^{\prime \prime}{ }_{0,0}+P_{0} 0_{C\left(K_{1,0}\right)} \oplus I d_{Y_{+}} I_{0}=S^{\prime \prime}{ }_{0,0}+0_{C\left(K_{1,0}\right)} \oplus I d_{C\left(K_{2,0}\right)}
$$

Of course $P_{m} I_{n}$ is zero or the identity operator depending whether $m=n$ but in both cases it is a multiplication by a continuous function, also multiplying by 0 on $K_{1,0}$ and by 1 on $K_{2,0}$ is a multiplication by a continuous function, so by 5.1 all the operators $S_{m, n}^{\prime}$ and $S_{m, n}^{\prime \prime}$ are zero operators concluding the lemma. 
PIOTR KOSZMIDER

Corollary 5.10. Suppose that $T, R: X_{+} \rightarrow X_{+}$are such operators that $R \circ T=$ $0_{C\left(K_{1,0}\right)} \oplus I d_{Y_{+}}$and $T \circ R=I d_{X_{+}}$. Then we have the following:

- $\sum_{k \in \mathbb{N}}\left(\phi_{m, k}^{R} \circ i_{k, n}\right) \phi_{k, n}^{T}(x)=0$ for distinct $n, m \in \mathbb{N}$ and all $x \in K_{n}$,

- $\sum_{k \in \mathbb{N}}\left(\phi_{n, k}^{R} \circ i_{k, n}\right) \phi_{k, n}^{T}(x)=1$ for all $(n, x) \in\left(\mathbb{N} \backslash\{0\} \times K_{+} \backslash K_{0}\right) \cup\left(\{0\} \times K_{2,0}\right)$,

- $\sum_{k \in \mathbb{N}}\left(\phi_{0, k}^{R} \circ i_{k, 0}\right) \phi_{k, 0}^{T}(x)=0$ for all $x \in K_{1,0}$,

- $\sum_{k \in \mathbb{N}}\left(\phi_{m, k}^{T} \circ i_{k, n}\right) \phi_{k, n}^{R}(x)=0$ for distinct $n, m \in \mathbb{N}$ and all $x \in K_{n}$,

- $\sum_{k \in \mathbb{N}}\left(\phi_{n, k}^{T} \circ i_{k, n}\right) \phi_{k, n}^{R}(x)=1$ for all $n \in \mathbb{N}$ and all $x \in K_{n}$.

Lemma 5.11. Let $x \in K$ and $x_{n}=i_{n, *}(x)$ and $A=\left\{x_{n}: n \in \mathbb{N}\right\}$. Let $T: X_{+} \rightarrow$ $X_{+}$be a bounded linear operator. There is a bounded linear operator $\Pi_{x}^{T}: c_{0}(A) \rightarrow$ $c_{0}(A)$ which satisfies

$$
\Pi_{x}^{T}\left(1_{x_{n}}\right)\left(x_{m}\right)=\phi_{m, n}^{T}\left(x_{n}\right),
$$

and whose norm is not bigger than $\|T\|$.

Proof. Note that if $\overrightarrow{1}_{n}$ denotes the constant function on $K_{n}$ whose value is one, then $\Pi^{T}\left(\overrightarrow{1}_{n}\right)\left(x_{m}\right)=\phi_{m, n}^{T}\left(x_{n}\right)$. Since the range of $\Pi^{T}$ is $X_{0}$ we may conclude that $\left(\phi_{m, n}^{T}\left(x_{n}\right)\right)_{m \in \mathbb{N}}$ belongs to $c_{0}(A)$ and its norm is not bigger than $\|T\|$ by 5.6. So the above formula well defines $\Pi_{x}^{T}: c_{0}(A) \rightarrow c_{0}(A)$.

Lemma 5.12. Suppose that $T, R: X_{+} \rightarrow X_{+}$are such operators that $R \circ T=$ $0_{C\left(K_{1,0}\right)} \oplus I d_{Y_{+}}$and $T \circ R=I d_{X_{+}}$. Let $x \in K_{1, *}, y \in K_{2, *}, x_{n}=i_{n, *}(x) y_{n}=$ $i_{n, *}(y), A=\left\{x_{n}: n \in \mathbb{N}\right\}, A^{\prime}=\left\{x_{n}: n \in \mathbb{N} \backslash\{0\}\right\}, B=\left\{y_{n}: n \in \mathbb{N}\right\}$. Let

$$
\begin{aligned}
& \Pi_{x, y}^{T}=\left(\Pi_{x}^{T}, \Pi_{y}^{T}\right): c_{0}(A \cup B) \rightarrow c_{0}(A \cup B), \\
& \Pi_{x, y}^{R}=\left(\Pi_{x}^{R}, \Pi_{y}^{R}\right): c_{0}(A \cup B) \rightarrow c_{0}(A \cup B) .
\end{aligned}
$$

Then $\Pi_{x, y}^{R} \circ \Pi_{x, y}^{T}=0_{x_{0}} \oplus I d_{c_{0}\left(A^{\prime} \cup B\right)}$ and $\Pi_{x, y}^{T} \circ \Pi_{x, y}^{R}=I d_{c_{0}(A \cup B)}$.

Proof. Apply corollary 5.10 pointwise.

\section{Detecting Shifts in ISOMORPHiSms.}

If $I$ is a set and $f \in c_{0}(I)$, by support of $f$ we mean $\{i: f(i) \neq 0\}$.

Lemma 6.1. Let $A=\left\{x_{n}: n \in N\right\}$ and $B=\left\{y_{n}: n \in N\right\}$. Suppose that $T$ : $c_{0}(A \cup B) \rightarrow c_{0}(A \cup B)$ is an operator such that $T\left(1_{x_{0}}\right)=0$, such that $T \mid\left(\left\{f \in c_{0}(A)\right.\right.$ : $f(0)=0\})$ is an isomorphism onto $c_{0}(A)$ and $T_{2}=T \mid\left(c_{0}(B)\right.$ is an isomorphism onto $c_{0}(B)$. Then there are $f_{k} \in c_{0}(A \cup B)$ of pairwise disjoint and finite supports and there is an $\varepsilon>0$ for which

$$
f_{k}\left(x_{n}\right)=f_{k}\left(y_{n}\right)
$$

for every $n \in N$ and for some distinct $m_{k} s$ we have

$$
\left|T\left(f_{k}\right)\left(x_{m_{k}}\right)-T\left(f_{k}\right)\left(y_{m_{k}}\right)\right|>\varepsilon .
$$

Proof. Let $T_{1}=T \mid c_{0}(A)$. Consider $T_{3}: c_{0}(B) \rightarrow c_{0}(B)$ so that we have $T_{3}\left(1_{\left\{y_{n}\right\}}\right)\left(y_{m}\right)=$ $T_{1}\left(1_{\left\{x_{n}\right\}}\right)\left(x_{m}\right)$ for every $m, n \in N$. That is we consider a copy of $T_{1}$ copied from $c_{0}(A)$ on $c_{0}(B)$.

Claim: $T_{3}-T_{2}: c_{0}(B) \rightarrow c_{0}(B)$ is not weakly compact. 
Proof of the Claim: If it is, then it is strictly singular by 2.7, and so we will be able to apply the Fredholm theory (see [18]). Namely, then, the Fredholm index of $T=\left(T_{1}, T_{2}\right): c_{0}(A \cup B) \rightarrow c_{0}(A \cup B)$ is equal to the Fredholm index of $\left(T_{1}, T_{2}\right)+\left(0, T_{3}-T_{2}\right)=\left(T_{1}, T_{3}\right)$ which must be an even integer since $T_{1}$ and $T_{3}$ are copies of the same operator. However $T_{2}$ is an isomorphism and $T_{1}$ is onto and has kernel of dimension one, and so $T=\left(T_{1}, T_{2}\right)$ must have odd Fredholm index, a contradiction which completes the proof of the claim.

Now use 2.8 to find a pairwise disjoint $e_{k} \in c_{0}(B)$ such that there are $m_{k}$ s such that

$$
\left|\left[\left(T_{3}-T_{2}\right)\left(e_{k}\right)\right]\left(y_{m_{k}}\right)\right|>\varepsilon
$$

for some $\varepsilon>0$. Since the operator is bounded and the norms of sums of $e_{k}$ s are bounded as well since there are disjoint, we may assume that all $m_{k} \mathrm{~s}$ are distinct. Now define $f_{k} \in c_{0}(A \cup B)$ by $f_{k} \mid B=e_{k}$ and $f_{k}\left(x_{n}\right)=e_{k}\left(y_{n}\right)$ for each $n \in N$. So we have $f_{k}\left(x_{n}\right)=f_{k}\left(y_{n}\right)$ for each $k, n \in N$. Also

$$
\begin{gathered}
\left.T\left(f_{k}\right)\left(x_{m_{k}}\right)=T_{1}\left(f_{k} \mid A\right)\left(x_{m_{k}}\right)=T_{3}\left(e_{k}\right)\right)\left(y_{m_{k}}\right), \\
\left.T\left(f_{k}\right)\left(y_{m_{k}}\right)=T_{2}\left(e_{k}\right)\right)\left(y_{m_{k}}\right)
\end{gathered}
$$

so we obtain

$$
\left|T\left(f_{k}\right)\left(x_{m_{k}}\right)-T\left(f_{k}\right)\left(y_{m_{k}}\right)\right|>\varepsilon
$$

as required.

Lemma 6.2. Suppose that $S: C\left(K_{n}\right) \rightarrow C\left(K_{m}\right)$ is a weakly compact operator. Let $\tau_{n}: K_{n} \rightarrow L_{n}$ be the canonical surjection as in Section 3. Then for each $j \in \mathbb{N}$

$$
\Gamma(S, j)=\left\{t \in L_{n}: \exists x \in \tau_{n}^{-1}(\{t\}) \quad\left|S^{*}\left(\delta_{i_{m, n}(x)}\right)\left(\tau_{n}^{-1}(\{t\})\right)\right|>1 / j\right\}
$$

is finite.

Proof. If $\Gamma(S, j) \subseteq L_{n}$ is infinite, we can choose a discrete sequence $\left\{t_{l}: l \in \mathbb{N}\right\} \subseteq$ $\Gamma(S, j)$. Let $x_{l} \in K_{n}$ be such that $\tau_{n}\left(x_{l}\right)=t_{l}$ and $\left|S^{*}\left(\delta_{i_{m, n}\left(x_{l}\right)}\right)\left(\tau_{n}^{-1}\left(\left\{t_{l}\right\}\right)\right)\right|>1 / j$. Now use the fact that

$$
\tau^{-1}[\{t\}]=\bigcap\left\{\tau^{-1}([a]): t \in[a], a \in C_{n}\right\}
$$

and the family whose intersection appears above is directed to conclude that for each $l \in \mathbb{N}$ there is an $a_{l} \in C_{n}$ such that $t_{l} \in\left[a_{l}\right]$ and $\left|S^{*}\left(\delta_{i_{m, n}\left(x_{l}\right)}\right)\left(\tau_{n}^{-1}\left[\left[a_{l}\right]\right]\right)\right|>1 / j$. Moreover, as $\left\{t_{l}: l \in \mathbb{N}\right\}$ is discrete we may w.l.o.g. assume that $a_{l}$ 's are pairwise disjoint. This means that

$$
\left|S\left(\chi_{\tau_{n}^{-1}\left[\left[a_{l}\right]\right]}\right)\left(i_{m, n}\left(x_{l}\right)\right)\right|>1 / j
$$

for each $l \in \mathbb{N}$, which contradicts 2.8 since $a_{l}$ 's are pairwise disjoint.

Theorem 6.3. $Y_{+}$and $X_{+}$are not isomorphic.

Proof. Suppose $T_{1}: Y_{+} \rightarrow X_{+}$and $R_{1}: X_{+} \rightarrow Y_{+}$be mutually inverse isomorphisms. Define $T, R: X_{+} \rightarrow X_{+}$by $R=R_{1}$ and $T=0_{C\left(K_{1,0}\right)} \oplus T_{1}$. We have $R \circ T=0_{C\left(K_{1,0}\right)} \oplus I d_{Y_{+}}$and $T \circ R=I d_{X_{+}}$.

By $5.12 \Pi_{x, y}^{T}$ satisfies the hypothesis of 6.1 To use it successfully we still need to make appropriate choice of $x$ and $y$. Let

$$
\Gamma=\left\{t \in L: \exists n, m, j \in \mathbb{N} h_{n, *}(t) \in \Gamma\left(S_{m, n}, j\right)\right\} .
$$


By $6.2 \Gamma$ is countable where $S_{m, n}=S_{m, n}^{T}$. Pick $t \in L \backslash \Gamma$ and $x \in K_{1}, y \in K_{2}$ such that $\tau(x)=\tau(y)=t$ (see 3.3). As usual let $x_{n}=i_{n, *}(x)$ and $y_{n}=i_{n, *}(y)$, and $t_{n}=h_{n, *}(t)$

Now use 6.1 obtaining $\alpha_{k} \in c_{0}(A \cup B)$ of pairwise disjoint and finite supports $F_{k}$ and an $\varepsilon>0$ such that $\alpha_{k}\left(x_{n}\right)=\alpha_{k}\left(y_{n}\right)$ for every $n \in N$ and for some distinct $m_{k} \mathrm{~s}$

$$
\left|\Pi_{x, y}^{T}\left(\alpha_{k}\right)\left(x_{m_{k}}\right)-\Pi_{x, y}^{T}\left(\alpha_{k}\right)\left(y_{m_{k}}\right)\right|>\varepsilon .
$$

For $n \in F_{k}$ we will find $a_{n} \in C_{n}$ such that the operator $T$ will have approximately the same behavior on vectors

$$
f_{k}=\sum_{n \in F_{k}} \alpha_{k}\left(x_{n}\right) \chi_{\tau_{n}^{-1}\left[\left[a_{n}\right]\right]} \in X_{0}
$$

as $\Pi_{x, y}^{T}$ on $\alpha_{k}$ s.

Note that for each $z_{n}$ satisfying $\tau\left(z_{n}\right)=t_{n}$ (in particular $z_{n}=x_{n}, y_{n}$ ), by the choice of $t$ from outside $\Gamma$, the measure $S_{m, n}^{*}\left(\delta_{i_{m, n}\left(z_{n}\right)}\right)$ of the set $\tau_{n}^{-1}\left[\left\{t_{n}\right\}\right]$ is zero. So, using the fact that $\tau_{n}^{-1}\left[\left\{t_{n}\right\}\right]=\bigcap\left\{\tau^{-1}([a]): t_{n} \in[a] \quad a \in C_{n}\right\}$ for each $n \in F_{k}$ find $a_{n} \in C_{n}$ such that $t_{n} \in\left[a_{n}\right]$ and

$$
\left|S_{m_{k}, n}^{*}\left(\delta_{x_{m_{k}}}\right)\left(\tau_{n}^{-1}\left(\left[a_{n}\right]\right)\right)\right|,\left|S_{m_{k}, n}^{*}\left(\delta_{y_{m_{k}}}\right)\left(\tau_{n}^{-1}\left(\left[a_{n}\right]\right)\right)\right|<\frac{\varepsilon}{3\left|F_{k}\right|\left|\alpha_{k}\left(x_{n}\right)\right|}
$$

so, for each $k \in \mathbb{N}$ and $n \in F_{k}$ we have

$$
\left|\alpha_{k}\left(x_{n}\right) S_{m_{k}, n}\left(\chi_{\tau_{n}^{-1}\left[\left[a_{n}\right]\right]}\right)\left(x_{m_{k}}\right)\right|,\left|\alpha_{k}\left(x_{n}\right) S_{m_{k}, n}\left(\chi_{\tau_{n}^{-1}\left[\left[a_{n}\right]\right]}\right)\left(y_{m_{k}}\right)\right|<\frac{\varepsilon}{3\left|F_{k}\right|}
$$

and so

$$
\mid S_{m_{k}, n}\left(\sum _ { n \in F _ { k } } \alpha _ { k } ( x _ { n } ) \chi _ { \tau _ { n } ^ { - 1 } [ [ a _ { n } ] ] } ( x _ { m _ { k } } ) | , | S _ { m _ { k } , n } \left(\sum_{n \in F_{k}} \alpha_{k}\left(x_{n}\right) \chi_{\tau_{n}^{-1}\left[\left[a_{n}\right]\right]}\left(y_{m_{k}}\right) \mid<\frac{\varepsilon}{3}\right.\right.
$$

and finally

$$
\left|S_{m_{k}, n}\left(f_{k}\right)\left(x_{m_{k}}\right)\right|,\left|S_{m_{k}, n}\left(f_{k}\right)\left(y_{m_{k}}\right)\right|<\varepsilon / 3
$$

for each $k \in \mathbb{N}$ and each $n \in F_{k}$. On the other hand

$$
\left|\Pi^{T}\left(f_{k}\right)\left(x_{m_{k}}\right)-\Pi^{T}\left(f_{k}\right)\left(y_{m_{k}}\right)\right|=\left|\Pi_{x, y}^{T}\left(f_{k}\right)\left(x_{m_{k}}\right)-\Pi_{x, y}^{T}\left(f_{k}\right)\left(y_{m_{k}}\right)\right|>\varepsilon
$$

hence for each $k$ we have

$$
\left|T\left(f_{k}\right)\left(x_{m_{k}}\right)-T\left(f_{k}\right)\left(y_{m_{k}}\right)\right|>\varepsilon / 3
$$

where $m_{k}$ s are distinct and $f_{k}$ 's are bounded $\left(C_{n}\right)$-simple and of disjoint supports.

Now note that for any infinite $M \subseteq \mathbb{N}$ there is in $X_{+}$the supremum $f_{M}$ of $\left\{f_{k}: k \in \mathbb{N}\right\}$.

Let $\zeta_{k}=T^{*}\left(\delta_{x_{m_{k}}}-\delta_{y_{m_{k}}}\right)$. In particular we have $\left|\zeta_{k}\left(f_{k}\right)\right|>\varepsilon / 3$. Apply 2.10 to obtain an infinite $M$ such that $\left|\zeta_{k}\left(f_{M}\right)\right|>\varepsilon / 4$ for each $k \in M$. But this means that

$$
\left|T\left(f_{M}\right)\left(x_{m_{k}}\right)-T\left(f_{M}\right)\left(y_{m_{k}}\right)\right|>\varepsilon / 4
$$

for each $k \in M$. This is the final contradictions with 3.10 since $x_{m_{k}} \bowtie y_{m_{k}}$.

Corollary 6.4. $X_{+}$and $Y_{+}$are not isomorphic but each is isomorphic to a complemented subspace of the other. 


\section{REFERENCES}

1. Cz. Bessaga, A. Pełczyński, On bases and unconditional convergence of series in Banach spaces. Studia Math. 171958 151-164.

2. J. Diestel, J.J. Jr. Uhl, Vector measures. Mathematical Surveys, No. 15. American Mathematical Society, Providence, R.I., 1977.

3. P. Casazza, The Schroeder-Bernstein property for Banach spaces. Contemp. Math., No. 15. (1989), 61-78

4. L. Drewnowski, An extension of a theorem of Rosenthal on operators acting from $l_{\infty}(G)$. Studia Math. 57 (1976), no. 3, pp. 209-215

5. R. Fajardo, An indecomposable Banach space of continuous functions which has small density. Fund. Math. 202 (2009), no. 1, pp. 43-63.

6. E. Medina Galego, Banach spaces complemented in each other without isomorphic finite sums. Bull. Pol. Acad. Sci. Math. 50 (1) (2002) pp. 1-9.

7. E. Medina Galego, On pairs of Banach spaces which are isomorphic to complemented subspaces of each other. Colloq. Math. 101 (2) (2004) pp. 279-287

8. E. Medina Galego, The Schroeder-Bernstein index for Banach spaces. Studia Math. 164 (1) (2004) pp. 29-38

9. E. Medina Galego, V. Ferenczi, Some Results on the Schroeder-Bernstein Property for Separable Banach Spaces. Canad. J. Math. Vol. 59 (1), 2007 pp. 63-84

10. T. Gowers, A solution to Banach's hyperplane problem. Bull. London Math. Soc. 26 (1994), pp. 523-530.

11. T. Gowers, A Solution to the Schroeder-Bernstein Problem for Banach Spaces. Bulletin of the London Mathematical Society 1996 28(3), pp. 297-304;

12. T. Gowers, B. Maurey, The unconditional basic sequence problem. Journal A. M. S. 6 (1993), pp. 851-874.

13. T. Gowers, B. Maurey, Banach spaces with small spaces of operators. Math. Ann. 307 (1997) pp. $543-568$.

14. S. Koppelberg, Handbook of Boolean algebras Vol 1. North-Holland 1989.

15. P. Koszmider, Banach spaces of continuous functions with few operators. Math. Ann. 330 (2004), pp. 151-183.

16. P. Koszmider, M. Martín, J. Merí, Isometries on extremely non-complex Banach spaces. Preprint 2009.

17. P. Koszmider, M. Martín, J. Merí, Extremely non-complex $C(K)$ spaces. J. Math. Anal. Appl. 350 (2009), pp. 601-615.

18. J. Lindenstrauss, L. Tzafriri, Classical Banach spaces. I. Sequence spaces. Springer-Verlag, Berlin-New York, 1977.

19. A. Pełczyński, Projections in certain Banach spaces. Studia Math. 19 (1960) pp. 209-227

20. A. Pełczyński, On strictly singular and strictly cosingular operators. I. Strictly singular and strictly cosingular operators in C(S)-spaces. Bull. Acad. Pol. Sci. 13, 1, 1965, pp. 31 - 37.

21. G. Plebanek, A construction of a Banach space $C(K)$ with few operators. Topology Appl. 143 (2004), pp. 217-239.

22. H. Rosenthal, On relatively disjoint families of measures, with some applications to Banach space theory. Studia Math. 371970 pp. 13-36.

23. W. Schachermeyer, On some classical measure-theoretic theorems for non-sigma-complete Boolean algebras. Dissertationes Math. CCXIV 1982.

24. I. Schlackow, Classes of $C(K)$ spaces with few operators D. Phil. Thesis. Univerity of Oxford, 2009, under the supervision of Prof. R. Haydon, available at http://people.maths.ox.ac.uk/schlack1/

25. I. Schlackow, Centripetal operators and Koszmider spaces Topology Appl. 155 (2008), no. 11, pp. $1227-1236$

Institute of Mathematics, Polish Academy of Sciences, ul. Śniadeckich 8, P.O. Box 21, 00-956, Warsaw, Poland

Instytut Matematyki Politechniki Łódzkiej, ul. Wólczańska 215, 90-924 Łódź, Poland 
Departamento de Análisis Matemático, Facultad de Ciencias, Universidad de Granada, 18071 Granada, Spain

E-mail address: piotr.math@gmail.com 